\title{
UN ACERCAMIENTO A LA CONSTRUCCIÓN DE LA CIRCUNCLISIS EN EL MAYA YUCATECO INFANTIL
}

\section{AN APPROACH TO THE CONSTRUCTION OF DISCONTINUITY IN EARLY YUCATEC MAYAN}

\author{
Mary Rosa Espinosa Ochoa
}

Universidad Nacional Autónoma de México, Instituto de Investigaciones Filológicas, Centro de Lingüística Hispánica Juan M. Lope Blanch

RESUMEN

En este trabajo de investigación se explora el proceso de adquisición del fenómeno sintáctico de la circunclisis en el maya yucateco infantil. Se analiza el habla espontánea de 6 adultos y 4 niños $(1 ; 02-3 ; 05)$ que adquirieron el maya yucateco en contextos monolingües. Concluimos que la circunclisis no es percibida como una unidad sintáctica en el inicio de la adquisición sino que requiere un desarrollo gradual en el que intervienen factores aunados a características tanto de la lengua maya yucateca (factores morfológicos, silábicos y prosódicos) como del habla adulta (omisión de elementos). Estos resultados apoyan la teoría de corte constructivista (Lieven, Behrens, Speares \& Tomasello, 2003). El texto que se presenta aquí constituye una versión actualizada de un capítulo de mi tesis de doctorado.

PALABRAS ClAVE: adquisición de lengua materna; maya yucateco; clíticos; deixis

Estudios de Lingüística Aplicada, año 35, número 66, diciembre de 2017, pp. 41-75 doi: 10.22201/enallt.01852647p.2017.66.682 
Abstract

This research explores the acquisition process of the discontinuous clitics in the syntax of Yucatec Mayan in child language. We analyzed the spontaneous speech of 6 adults and 4 children (1;02-3;05) who acquired Yucatec Mayan in a monolingual environment. We concluded that discontinuity is not perceived as a syntactic unit in the earliest acquisition stage. The full acquisition of discontinuous clitics is a gradual process that is influenced by several factors pertaining to the characteristics of the Mayan language and adult speech. These results support constructivist approaches in language acquisition (Lieven, Behrens, Speares \& Tomasello, 2003). This paper is an updated version of a chapter of my Phd thesis.

KEYWORDS: child language acquisition; Yucatec Mayan; clitics; deixis 


\section{INTRODUCCIÓN}

\subsection{El sistema deíctico del maya yucateco}

Los deícticos en maya yucateco resultan muy interesantes desde un punto de vista gramatical, ya que la mayor parte de ellos están compuestos por dos morfemas: uno al que Hanks (1990) ha llamado base, que indica la categoría gramatical, y un enclítico o sufijo vocálico con cierre glotal, que contiene casi siempre la mayor parte de la información deíctica. Algunos investigadores han optado por considerarlos clíticos pues indican su independencia semántica con respecto a elementos que les anteceden o suceden cuando llegan a introducirse otros afijos u otros elementos léxicos (Monforte, 1999: 102). De aquí en adelante se les denominará enclíticos. Estos expresan relaciones espaciales, perceptuales y modales en el sistema deíctico del maya yucateco. En el Cuadro 1 transcribo la sinopsis de este sistema de acuerdo con Hanks (1990: 18), la columna corresponde a las bases y la fila superior muestra los enclíticos.

Cuadro 1. Sinopsis de los deícticos en maya yucateco (Hanks, 1990: 18)

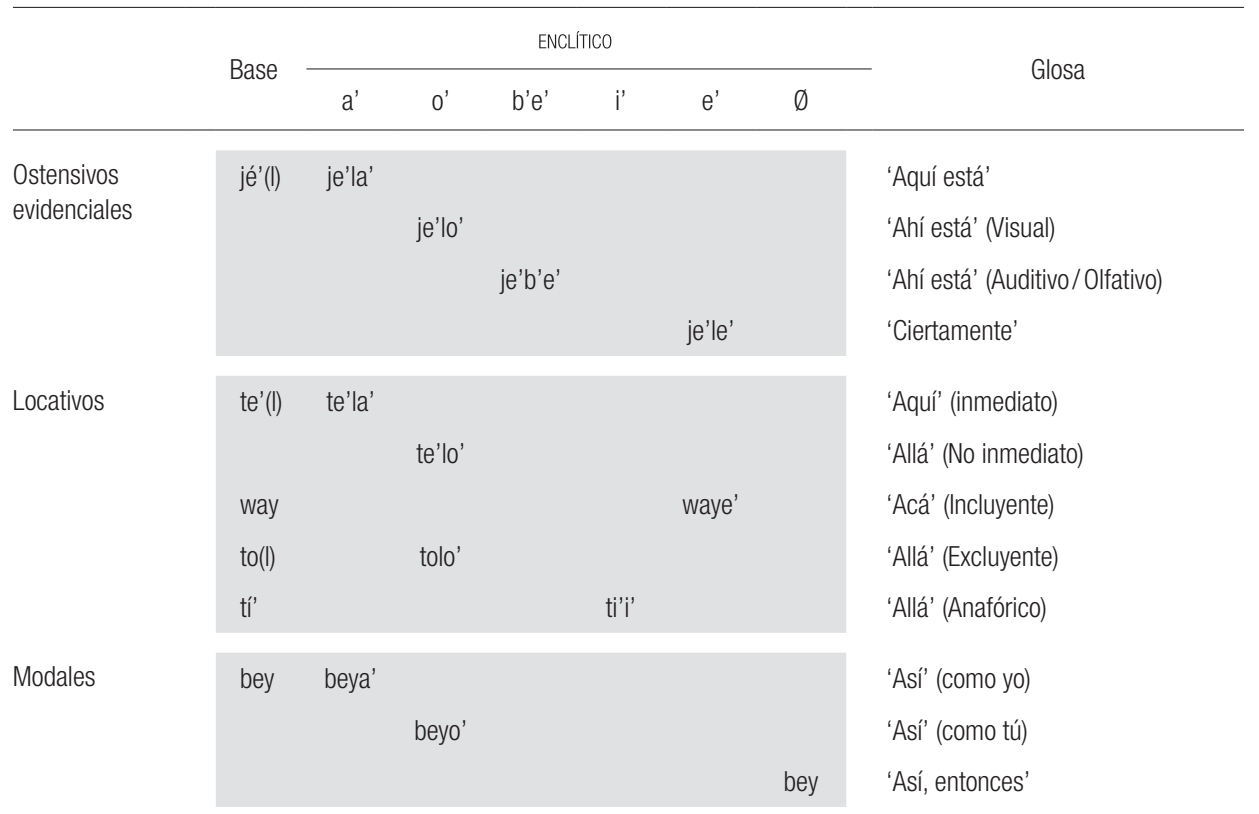

Estudios de Lingüística Aplicada, año 35, número 66, diciembre de 2017, pp. 41-75 doi: 10.22201/enallt.01852647p.2017.66.682 


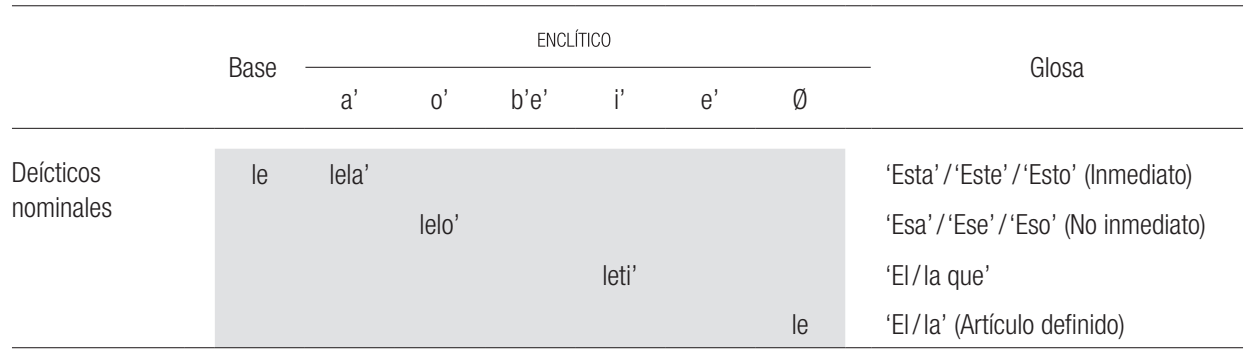

Las bases se combinan con los enclíticos, aunque existen restricciones. Los ostensivos evidenciales $j e$ ' $l$ - se coordinan con todos los enclíticos excepto - $t i$ ', y es el único que se combina con el auditivo/olfativo - $b e^{\prime}$. La base locativa adverbial $t e^{\prime}$ - se combina solo con $-a^{\prime}$ y $-o$ ', lo mismo que el modal bey-. Mientras que el adverbio way- se coordina solo con - $e^{\prime}$ y to'ol- solamente con -o'. El locativo focal tí' se combina solo en el anafórico - $t i^{\prime} i$. El demostrativo (deíctico nominal) le puede combinarse con todos excepto el topicalizador $-e$ '. Las bases contienen el rasgo de la categoría gramatical, como adverbios, determinantes, pronombres, y por tanto determinan el rol sintáctico dentro de la oración (argumento, circunstancial, etcétera). Asimismo, codifican ciertas diferencias abstractas de significado entre ellas tal como presentación ostensiva (je'), egocéntrico incluyente (way) y excluyente (tol), sociocéntrico (te'), e incluso los participantes de la interlocución como categoría (Hanks, 1990). Los enclíticos añaden al significado de las formas deícticas "a relatively subtle array of indexical values, including Assymmetric, new information (- $\left.a^{\prime}\right)$; Symmetric, preestablished information $\left(-o^{\prime}\right)$; Perceptible, but not in sight, which I have labeled 'peripheral sensory' (- $\left.b^{\prime} e^{\prime}\right)$ "' (Hanks, 1990: 58). ${ }^{1}$ Otros autores prefieren referirse a los valores semánticos que añaden los enclíticos desde su definición más tradicional como próximo (- $\left.a^{\prime}\right)$, distal o no marcado (-o’) (véase, por ejemplo, Bohnemeyer $\&$ Stolz, 2006). Aunque la mayoría de las veces, la oposición proporcional espacial de cada deíctico está dada por la relación paradigmática en que lo sitúan los

1 “[U]na relativa sutil colección de valores indicadores, que incluyen asimetría, información nueva $\left(-a^{\prime}\right)$; simetría, información preestablecida $\left(-o^{\prime}\right)$; perceptibilidad, aunque no visual, que yo he llamado 'periférico sensorial' (-b’e')." Las traducciones de las citas son mías.

Estudios de Lingüística Aplicada, año 35, número 66, diciembre de 2017, pp. 41-75 doi: 10.22201/enallt.01852647p.2017.66.682 
enclíticos, también a veces codifican dimensiones espaciales. Cada deíctico conformado por su base más un enclítico da como resultado un universo semántico propio. Por ejemplo en (1), la expresión: ${ }^{2}$

$\begin{array}{lll}\text { (1) Lela' } & & \\ \text { Le } & \text { (l) } & \text {-a' } \\ \text { DEM } & \text { EP } & \text { ENCL PROX } \\ \text { 'Esto' } & & \end{array}$

Por la base le sabemos que es un pronombre demostrativo, aunque funciona también como artículo definido y marca la frase nominal (Monforte, 1999: 100) en construcciones discontinuas. El enclítico glotal $-a$ ' señala que el objeto se encuentra perceptual, espacial o temporalmente inmediato a la situación comunicativa del hablante con respecto al oyente, o bien anticipa el referente: "They are associated with high-focus gestures (presenting in hand, touching, pointing to a referent) referring to unique objects (as opposed to classes or vaguely known ones) with new (as opposed to already shared) information" (Hanks, 1990: 22). ${ }^{3}$ Como vemos en (1), entre la base le y el enclítico - $a$ ' se inserta una lateral que funciona como epentética, esto sucede en el maya yucateco en todos los casos en que la base tiene terminación vocálica y el enclítico es una vocal.

El fenómeno que atañe directamente a este trabajo es la llamada circunclisis o discontinuidad, a lo largo del texto se le llama con uno y otro término indiferentemente. Hanks (1990) describe esta característica de los deícticos yucatecos como la posibilidad combinatoria que tienen dentro de la oración como construcciones discontinuas en contraste con las continuas. El ejemplo (1) muestra una forma continua, pues las partículas se encuentran unidas para formar un solo elemento; en el caso de (2) y (3) se trata de ejemplos discontinuos, pues entre la base y el enclítico puede insertarse material léxico:

2 Para consultar las abreviaturas de las glosas, véase el Anexo.

3 "Están asociados con señas para obtener el foco de atención con alto grado de acercamiento al referente (tales como presentar con un gesto de mano, tocar o señalar el referente), que involucran objetos únicos (en contraste con el señalamiento de clases enteras o referentes vagamente conocidos) y nuevos (en oposición con la información compartida).” 
(2) Le tsíimina'

$\begin{array}{lll}\text { Le } & \text { tsíimin } & -\mathrm{a} \\ \text { DET } & \text { SUST.caballo } & \text { ENCL PROX }\end{array}$

'Este caballo'

(3) Le sak tsíimino'obo'

Le sak tsíimin $\quad$ o'ob - o'

DET ADJ.blanco SUST.caballo PL ENCL DIST

'Esos caballos blancos'

Cuando esto sucede desaparece la epéntesis mostrada en el ejemplo (1), y en el caso del demostrativo adquiere otra categoría gramatical, de demostrativo pronominal a determinante. Este fenómeno de la discontinuidad deíctica es propio de todas las construcciones deícticas.

Hanks (1990) lo representa como x [Deíctico Inicial o Base x Descripción léxica Deíctico Final o enclítico], la categoría gramatical dada por el deíctico inicial muestra que esta será la categoría gramatical de toda la frase. Gutiérrez Bravo (2015: 84) apunta que esta propiedad del maya yucateco "permite determinar con certeza que las cláusulas relativas sí están incrustadas dentro de la frase nominal encabezada por el núcleo".

\subsection{Un acercamiento a la adquisición del lenguaje infantil}

\subsubsection{Los determinantes}

Aunque este estudio tiene un acercamiento de corte constructivista podemos decir que, desde cualquier punto de vista teórico, la adquisición de los determinantes ha llamado la atención, pues su uso requiere diversas habilidades discursivas: el conocimiento del referente en foco, el tipo de sustantivo (en inglés, p. e. contable vs. no contable) y el grado de acuerdo en cuanto al tópico. Según Tomasello (2003: 207-208), una de las estrategias más importantes utilizadas por los niños más pequeños es el uso de una frase nominal (FN) que contiene algún tipo de sustantivo común y algún tipo de determinante. Estas frases nominales son producidas entre las primeras etapas de palabras múltiples, algunas veces como 
una oración completa en respuesta a una pregunta, p. e. Adulto: ¿Qué quieres? Niño: Mi payaso. Según Karmiloff Smith (1979) en la etapa temprana el uso de los artículos definidos e indefinidos no está relacionado entre sí. Este argumento se refuerza por los primeros estudios constructivistas que muestran que la adquisición de los determinantes en inglés es de base léxica. Pine y Martindale (1996) y Pine y Lieven (1997) realizaron estudios basados en observaciones de habla espontánea de niños cuya lengua materna era el inglés. Según Pine y Lieven (1997), los artículos definidos e indefinidos comienzan a utilizarse alrededor de los dos años, asociados a ítems de base léxica que aparecen inmediatamente después de ciertas preposiciones o verbos como se muestra enseguida:

- Artículos definidos: "Where's the X", "In/on the X", "There's the X" - Artículos indefinidos: "That's a X", "A X", "Get a X", "Want a X"

Pine y Martindale (1996) detectaron además que los niños usan el determinante definido con un grupo específico de sustantivos y el indefinido con otro grupo de sustantivos que casi no se superpone con el primero, a excepción de $10 \%$ de los sustantivos que los niños usan a los 2;01 que se traslapa con ambos artículos. Desde este enfoque, Tomasello (2003: 112-113) proporciona una sinopsis detallada sobre el proceso de adquisición de los determinantes con base en resultados obtenidos tanto de la experimentación como de la observación de discurso espontáneo: los niños pequeños comienzan usando artículos en distribución complementaria, es decir, sin traslape. En una etapa ligeramente posterior, antes del tercer año, comienzan a establecer la diferencia entre los artículos definidos e indefinidos con respecto solo a su especificidad frente al referente. Estas observaciones se insertan entre los principales puntos de partida de la teoría constructivista, a la que se adscribe este trabajo. Una investigación importante para sostener esta teoría fue realizada por Lieven, Behrens, Speares y Tomasello (2003). Ellos realizaron un análisis de datos de tipo espontáneo que contiene un seguimiento exhaustivo del habla de la niña Annie, angloparlante, en interacción con su madre entre los 2;00 y los 2;01,11. Analizaron todas las oraciones emitidas por la niña para estimar la posibilidad de que las cadenas de habla fueran aprendidas de raíz con especificidad léxica. De acuerdo con sus resultados solo $10 \%$ de sus oraciones con palabras múlti- 
ples fueron repetitivas dentro del corpus (oraciones que la niña había dicho antes) y aproximadamente la mitad de ellas fueron novedosas; en un 15\% de las oraciones la novedad consistía únicamente en que una palabra de frecuencia alta, como por ejemplo un deíctico, era añadida; y $26 \%$ de las oraciones fueron reacomodadas por los investigadores en una o dos operaciones para obtener la oración producida por la niña. Es decir, lo creativo en este último $26 \%$ de las oraciones consistía en el reacomodo de las oraciones ya conocidas por la niña. Las operaciones usadas por Annie fueron de sustitución de palabras dentro de la oración ya conocida, adición, inserción y reacomodo. Lo anterior prueba que la gramática no surge repentinamente, sino que se construye de forma paulatina con base en el discurso primario que la niña fue formando en sus dos primeros años de vida.

Esta construcción paulatina parte de ítems léxicos, construcciones adquiridas con base en el uso, directamente influenciadas por el habla que el niño escucha. En las culturas euroamericanas esta ha sido identificada como el habla dirigida al niño. Se ha probado que las bases léxicas que dan fundamento al proceso de adquisición son las construcciones más frecuentes en el habla dirigida al niño. Por ejemplo, Theakston, Lieven, Pine y Rowland (2002) investigaron la adquisición de la estructura argumental aunada a la construcción del paradigma verbal del verbo go. Sus resultados apuntan a que las estructuras sintácticas y semánticas de este verbo reflejan el patrón de adquisición del verbo en cuestión. En esta misma línea, Espinosa Ochoa (2011) detectó para el español que la construcción de base léxica más frecuente y primaria en el total de las interrogativas producidas por un niño hispanohablante era también la construcción más frecuente en el input. Se sabe, asimismo, que en la adquisición infantil, como en cambio lingüístico, la frecuencia de ocurrencias asegura el uso y la comprensión de piezas concretas de lenguaje (Bybee, 1995). La frecuencia de tipos, en cambio, provee al niño generalizaciones que le muestran que una misma construcción en diferentes contextos sirve para diferentes propósitos (Lieven, 2008). Aunque el habla dirigida al niño no es una práctica natural en todas las culturas, incluyendo la que atañe a este estudio, existen investigaciones que muestran el tipo de interacción que integra a los niños como miembros de la sociedad. ${ }^{4}$ Este

\footnotetext{
${ }^{4}$ Para consultar detalles sobre la interacción con niños en maya yucateco, véase Pfeiler (2007).
} 
proceso de integración apoya, por medio de la socialización, el desarrollo del lenguaje (para lenguas mayas véase p. e. De León, 2012). Pye, Pfeiler, De León, Brown y Mateo (2007) muestran que la frecuencia de determinados patrones en el habla adulta tiene un impacto en los modelos de adquisición infantil de las lenguas mayas. A su estudio nos referimos en el siguiente apartado.

\subsubsection{El estado de la cuestión en la adquisición del maya yucateco}

Pfeiler (2002) documentó que la adquisición de la morfología en yucateco comienza con el uso de los sufijos deícticos, incluyendo al topicalizador - $e$ ', mismo que, por supuesto, caracteriza la aparición de la morfología nominal, seguido por sufijos de plural con sustantivos animados y en tercer lugar por prefijos posesivos. Según el estudio de Flores Vera (1998), los deícticos son una categoría léxica muy frecuente en la adquisición temprana del maya yucateco, sobre todo entre $1 ; 01$ y 1;04, incluso más que en habla adulta. Documenta como la entrada léxica más usada el tipo je’le', que el niño utiliza como "deíctico distante, 'allí', como afirmativo 'sî' o 'así es', como demostrativo 'aquello/a' e incluso como deíctico visual 'mira"' (Flores, 1998: 81). En cuanto a la adquisición de la morfología verbal del maya yucateco, Carrillo Carreón (2005) encontró un proceso de adquisición de corte constructivista, en que la estrategia de adquisición de la ergatividad de modelo mixto se cuelga de una serie limitada de verbos de uso frecuente cuya morfología se extiende poco a poco a verbos de uso más escaso.

Con un acercamiento un tanto distinto, contamos también con los estudios comparativos sobre la morfología verbal de lenguas mayas (Brown, 1997, 1998; De León, 1999; Pfeiler \& Briceño, 1997; Pye, 1983). Los investigadores apuntan que los niños que aprenden k'iche', tzeltal, tzotzil, q'anjob'al y yucateco producen formas diferentes en sus primeros verbos.

Los niños que adquieren tzeltal y tzotzil en una etapa inicial producen una proporción muy alta de raíces verbales, mientras que los niños aprendices de k'iche', q'anjob'al y yucateco producen bases verbales con sufijos (Pye et al., 2007). La frecuencia con que los adultos producen raíces verbales a la derecha de las palabras y oraciones influye en la frecuencia de producción verbal infantil, pero, en cambio, la frecuencia con que se producen a la izquierda no repercute en la producción. "The position that the verb usually occupies in the sen- 
tence (initial position) does not affect children's ability to extract the verb root, nor does the degree to which the verb carries inflectional prefixes" (Pye et al., 2007: 40). ${ }^{5}$ Su objetivo era examinar la relación entre la dimensión de la complejidad lingüística y su velocidad en el desarrollo lingüístico infantil. Según sus resultados, la velocidad de adquisición en el habla infantil es más alta en la morfología verbal que en la nominal, lo que corresponde sistemáticamente con el grado de mayor riqueza en la morfología verbal.

En un estudio comparativo entre la adquisición del español y el maya yucateco, Espinosa Ochoa (2007) documenta que los determinantes aparecen en etapas más tempranas en la primera lengua que en la segunda. En particular, los niños maya hablantes no producen la base en posición de oración final, aunque sí producen los enclíticos. Este fenómeno se atribuye a la influencia de diversos factores propios de la naturaleza de cada lengua. En español, los pronombres aparecen en etapas muy tempranas (Espinosa Ochoa, 2002) y estos funcionan como soporte para la adquisición de los determinantes que son categorías léxicas, por ejemplo, el hecho de que los niños estén utilizando el pronombre 'este', los ayudará a producir posteriormente una frase como 'este conejo'. En esta lengua hay una tendencia a producir con mayor velocidad múltiples palabras. En maya yucateco, en cambio, los niños deben dominar la morfología antes de producir multiplicidad de palabras, debido a la riqueza morfológica de la lengua (Aksu Koç \& Slobin, 1985). Por otro lado, en el habla adulta la base de los determinantes es oscura cuando estos aparecen en oraciones finales, lo cual puede estar posponiendo la aparición de la base le. Cabe mencionar que los niños mayas más jóvenes del estudio sí produjeron los enclíticos.

Resultados parecidos han sido documentados por Pye (1992) en la adquisición del maya quiché, en esta lengua los niños tienden a producir de forma más temprana los elementos que están al final de oración.

5 "La posición que generalmente ocupa el verbo en la oración (posición inicial) no afecta la capacidad de los niños para extraer la raíz del verbo, ni tampoco el grado en que el verbo lleva los prefijos inflexionales." 


\section{OBJETIVOS DE INVESTIGACIÓN}

En este estudio trataremos precisamente de observar cómo los niños en diferentes etapas del desarrollo lingüístico usan estas palabras, así como en qué contextos y circunstancias, para así proponer cómo se adquiere la circunclisis. Se han formulado las siguientes preguntas específicas de investigación:

a) ¿Se manifiestan etapas de desarrollo de la circunclisis? ¿Reconocen los niños ambos elementos y desarrollan a la par la continuidad y discontinuidad de estos clíticos?

b) ¿Existe alguna tendencia durante la adquisición hacia la producción de la base (lado izquierdo del sustantivo) o del enclítico (lado derecho)?

\section{Metodología}

Para la elaboración de este trabajo se utilizaron dos bases de datos longitudinales de emisiones espontáneas y una base transversal de datos también espontáneos, aunque inducidos por medio de juegos que suscitan la emisión de deícticos.

\subsection{Bases de datos longitudinales}

\subsubsection{Campamento Hidalgo}

Este corpus está constituido por datos de corte longitudinal que se recolectaron durante un año desde octubre de 2006 hasta octubre de 2007 en la comunidad de Campamento Hidalgo, Quintana Roo. Las edades de los niños y las fechas de recolección pueden verse en el Cuadro 2. Estos datos fueron recolectados por Silvestre Naal Llanez, hablante nativo del maya yucateco, quien visitó esta comunidad durante un año una vez al mes. Sus visitas no fueron estrictamente cada 30 días, sino aproximadamente cada 23 o 37 días. Incluso, entre la toma de septiembre y octubre, hay una diferencia de casi 50 días. $^{6}$

6 La videograbación de julio permaneció en la cámara todo un mes, esto le provocó hongos debido al ambiente de humedad de la península yucateca y por tanto no pudo ser utilizada en la muestra.

Estudios de Lingüística Aplicada, año 35, número 66, diciembre de 2017, pp. 41-75 doi: 10.22201/enallt.01852647p.2017.66.682 
52 Mary Rosa Espinosa Ochoa

\begin{tabular}{clcc}
\multicolumn{4}{c}{ CuAdRo 2. Edades de los niños durante la obtención de la base de datos longitudinal de Campamento Hidalgo } \\
\hline No. DE VIDEO & FECHA DE REALZACIÓN & EdAD FEL & EDAD VAL \\
\hline 1 & Octubre, 2006 & ------ & $3 ; 08,01$ \\
2 & Noviembre, 2006 & ------ & $3 ; 09,01$ \\
3 & Diciembre, 2006 & ----- & $3 ; 09,28$ \\
& Enero, 2007 & ----- & $3 ; 11,00$ \\
& Febrero, 2007 & ------ & $3 ; 11,29$ \\
4 & Marzo, 2007 & $2 ; 10,07$ & $4 ; 00,27$ \\
5 & Abril, 2007 & $2 ; 11,13$ & $4 ; 02,01$ \\
6 & Mayo, 2007 & $3 ; 00,02$ & $4 ; 02,22$ \\
7 & Junio, 2007 & $3 ; 01,00$ & $4 ; 03,20$ \\
8 & Agosto, 2007 & $3 ; 03,01$ & $4 ; 05,25$ \\
9 & Septiembre, 2007 & $3 ; 03,29$ & $4 ; 06,19$ \\
10 & Octubre, 2007 & $3 ; 05,24$ & $4 ; 08,14$
\end{tabular}

Para promover que los niños hablaran desde el principio de la grabación, se comenzaba con juguetes que proporcionaba el observador: dos marionetas de peluche, un cubo con tapa de figuras geométricas, diez cubos rompecabezas, dos libros con historias ilustradas y tarjetas con dibujos. Aunque las grabaciones comenzaron casi siempre con estos juguetes, después de 20 minutos o media hora los niños cambiaban el juego, mismo que se efectuaba con los objetos propios de su entorno.

\subsubsection{Yalcobá}

Se analizó también una pequeña parte de la muestra longitudinal de un niño primogénito de Yalcobá, Yucatán, a quien llamaremos ARM. Esta fue recabada por la Dra. Bárbara Pfeiler en el proyecto Las etapas pre- y proto-morfológicas en la adquisición de la lengua materna: el yucateco. ${ }^{7}$ Las audiograbaciones fueron realizadas por la responsable del proyecto con la colaboración de una maestra de primaria de la región; se utilizaron aproximadamente 12 horas de grabación en 18 transcripciones obtenidas en el proyecto. La transcripción y traducción al español estuvieron

\footnotetext{
7 Conacyt No. 4639-H.
}

Estudios de Lingüística Aplicada, año 35, número 66, diciembre de 2017, pp. 41-75 doi: 10.22201/enallt.01852647p.2017.66.682 
a cargo de dos hablantes nativos del yucateco y la captura de datos fue realizada por estudiantes de lingüística de la Universidad Autónoma de Yucatán. Para este trabajo se utilizaron 18 transcripciones obtenidas en el proyecto, correspondientes a las siguientes edades: $1 ; 02,23,1 ; 02,27,1 ; 03,07,1 ; 03,21,1 ; 05,06,1 ; 05,10$, $1 ; 05,19,1 ; 05,22,1 ; 06,03,1 ; 06,06,1 ; 06,10,1 ; 06,13,1 ; 06,20,1 ; 06,26,1 ; 07,01$, $1 ; 07,08,1 ; 07,10,1 ; 07,15$.

\subsection{Datos transversales: Yalcobá}

Los datos transversales se recolectaron también en el poblado de Yalcobá. Los datos obtenidos en esta muestra se centran en el estudio de dos niños, KEN $(2 ; 03)$ y DAN $(2 ; 07)$, a pesar de que otros niños de sus respectivas familias también participaron aquí. La muestra de Yalcobá consiste de ocho videograbaciones que contienen datos espontáneos pero que no fueron grabadas en escenas de vida cotidiana, sino mediante actividades lúdicas que por su naturaleza producen la emisión de deícticos. Los juegos elegidos para estas pruebas de tipo espontáneo fueron: un rompecabezas sencillo, apto para niños a partir de los 4 años, con piezas relativamente grandes; un rompecabezas complicado con piezas pequeñas, y Mega Blocks. Se buscaron juegos en los que se utilizaron numerosas piezas de formas similares — muy difícilmente referibles por medio de un nombre propio - cuyo fin fuera acomodar dichas piezas en algún lugar específico, circunstancia que motivara la necesidad de que los interlocutores las señalaran. Como los niños más pequeños, especialmente entre los 2 y los 4 años de edad, no podían interactuar fácilmente con estos juegos, se les videograbó también coloreando libros y tarjetas, actividades que propician de igual manera la emisión de los deícticos. Antes de empezar a grabar dos de los videos con niños pequeños, se comenzó con 20 minutos de juego de pelota, que, aunque no con la misma frecuencia, también propició el uso de deícticos.

En los videos los niños están agrupados de acuerdo al grado de familiaridad entre ellos y la locación es siempre su propio hogar o casas a las que están acostumbrados. Esto con el objetivo de que se sintieran en un ambiente de más confianza y más apegado a su entorno cotidiano.

En los dos grupos de adultos que accedieron amablemente a realizar esta tarea también se siguieron las condiciones para los niños anteriormente mencionadas. 
Entre los adultos teníamos contempladas inicialmente las cuatro personas voluntarias para armar las piezas de los rompecabezas y blocs, las cuatro de sexo femenino de edades de entre 23 y 65 años. Sin embargo, agregamos a este análisis los deícticos de dos adolescentes de 14 y 15 años, hombre y mujer, respectivamente, quienes participan de forma activa con uno de los niños.

Las transcripciones tanto del corpus longitudinal de Campamento Hidalgo como del corpus transversal de Yalcobá fueron revisadas con una hablante nativa del yucateco, las intenciones de los hablantes al usar los deícticos fueron también discutidas con ella. Las transcripciones se centraron en las emisiones de los deícticos y se consideró todo el contexto anterior o posterior necesario para comprender cada emisión. Se descartaron todas aquellas emisiones que resultaban ambiguas o ininteligibles para ella.

\section{ORGANIZACIÓN DE LOS DATOS}

En primer lugar se presentan los datos longitudinales de ARM, niño de Yalcobá, pues son los que arrojan datos más tempranos — las primeras emisiones desde el 1;02 hasta el 1;07. En segundo lugar mostramos los datos transversales de los niños, también provenientes de Yalcobá, KEN $(2 ; 03)$ y DAN $(2 ; 07)$, pues el habla de estos niños corresponde a una etapa más avanzada. Se presentan por último los datos longitudinales de FEL $(2 ; 07-3 ; 05)$, el niño de menor edad de nuestro corpus de Campamento Hidalgo, que nos permitieron explorar una etapa más avanzada en el desarrollo infantil temprano. Para cada niño se realizó el mismo análisis. Se recolectaron todos los deícticos, bases y enclíticos emitidos, se confrontaron los datos para encontrar analogías y diferencias entre las oraciones y detectar el desarrollo gradual que presentaran. El contraste se estableció tanto entre el desenvolvimiento detectado para cada niño, en los casos en que la naturaleza longitudinal de la base de datos lo permitía — es decir, únicamente para ARM y FEL-, como en contraste entre los niños (las oraciones de ARM en contraste con las de KEN, en contraste con las de DAN, en contraste con las de FEL). Este último contraste se llevó a cabo para conocer el desarrollo desde el 1;02 (edad en que se recogieron datos del niño más pequeño) hasta los 3;08 (edad en que se recogieron datos del niño más grande). 


\section{ANÁlisis DE LOS DATOS: LAS ETAPAS DEL DESARROLLO}

A continuación se presenta el marco gradual de desarrollo de los deícticos desde las emisiones continuas hacia la producción de la discontinuidad, haciendo énfasis únicamente en el análisis de los cambios sobresalientes que nos permitan describir el desarrollo.

\subsection{Primera etapa estudiada. El niño ARM $(1 ; 02,23-1 ; 07,15)$}

Los primeros ejemplos son de producciones ininteligibles similares a los presentados para el inglés por Johnson (1983: 113), tal como el que se presenta en (4):

(4) 'wh-thisonegomom' 'wh-disone'

En este tipo de emisiones no se distingue ninguna palabra ni sintaxis concreta, solo una repetición ambigua de una frase o palabra. En (5) transcribimos emisiones del maya yucateco encontrados en el corpus de ARM. Estas emisiones dejan entrever por primera vez la presencia primitiva de un deíctico; si bien los deícticos son realmente amorfos, el transcriptor los ha interpretado como tales.

(5) ARM: ya' (probablemente je'la')

'toma'

MAM: ¿tu'uxaan?

¿¿dónde está?'

ARM: hu', hu', da' (je', je', la' (i))

'aquí, aquí, está' [ARM, 1;01,12]

En los veinte días siguientes a las audiograbaciones anteriormente citadas, encontramos señales más claras de la presencia de un deíctico. Reproducimos dos ejemplos en (6).

(6) a. eela'

(j)e'-1-a' (i)

'aquí está' [ARM, 1;01,16]

Estudios de Lingüística Aplicada, año 35, número 66, diciembre de 2017, pp. 41-75 doi: 10.22201/enallt.01852647p.2017.66.682 


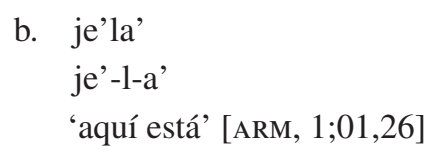

Hacia el 1;07,03 seguimos encontrando emisiones truncas (aféresis y apócopes) de los deícticos, como en (7).

(7) a. je'e (apócope de je'la') [ARM, 1;01,30]

b. be' (aféresis de je'be') [ARM, 1;02,16]

A la edad de 1;02,26 ARM comienza a combinar los deícticos, aunque no lo hace a la manera adulta, sino que produce una forma simultáneamente a la otra, como se aprecia en (8).

(8) la', je'lo', lo' (aféresis je'la', y je'lo')

'toma, mira, allí' [ARM, 1;02,26]

Encontramos también casos de construcción de una base con un pronombre personal, como en (9).

(9) je'e'éech

$=(j) e^{\prime}(l a ')$ teech $^{8}$

'este tú' [ARM, 1;02,26]

Al 1;07 encontramos finalmente una combinación completa, como se observa en (10).

(10) ma' lete-o'

$\begin{array}{lllll}\text { ma' } & \text { le } & \text { te } & \text { (l) } & - \text { o' } \\ \text { NEG } & \text { DEI } & \text { DEI } & \text { EP } & \text { ENCL }\end{array}$

'no, lo de allí' [ARM, 1;07]

8 Se decidió agregar el signo = a la glosa inferior de las oraciones infantiles para indicar la reconstrucción que la hablante nativa realizó para reproducir el habla adulta. 
A la edad de 1;02,23 ARM comienza a combinar las bases y los clíticos con sustantivos, de la misma manera en que combina los deícticos entre sí, en bloque, proceso que se prolonga hacia el final de los datos analizados; véase (11).

$$
\begin{aligned}
& \text { (11) te'la'...ba' } \\
& \text { te' }-1 \quad-a{ }^{\prime} \text { ba'al } \\
& \text { DEI EP ENCL SUST.cosa } \\
& \text { 'aquí cosa' [ARM, 1;02,23] }
\end{aligned}
$$

No se encontró ningún deíctico + sustantivo con la forma del habla adulta, como en (12).
(12) le che'
$-\mathrm{o}$
DET SUST-tronco ENCL

'Ese tronco'

Esto significa que no hay aún presencia clara del uso de la circunclisis en esta etapa. Los datos de ARM podrían estar mostrando construcciones congeladas, como lo vemos en (13), el único caso de circunclisis encontrado.

$$
\begin{aligned}
& \text { (13) le teo' } \\
& =\text { le te'(l)-o' } \\
& \text { le te' }-1 \text {-o' } \\
& \text { DEI DEI -EP -ENCL }
\end{aligned}
$$

'Ese de allá'

Sin embargo, no encontramos indicios de repetición de algún enclítico determinado aunado a algún elemento específico. Lo que sí revelan es la presencia de los enclíticos con algún elemento, las bases combinadas únicamente de forma continua: deícticos continuos con sustantivos y ergativos, como se puede ver en los ejemplos (8) a (11). 
5.2. Una etapa intermedia. KEN $(2 ; 04)$ y DAN $(2 ; 08)$

La siguiente base de datos analizada, por obedecer al orden cronológico, es la de Yalcobá, de tipo transversal. Dentro de este corpus se utilizaron únicamente los deícticos de KEN $(2 ; 04)$ y DAN $(2 ; 08)$. No se tomaron en cuenta los deícticos aislados, sino solo los encontrados en combinación con otras categorías gramaticales, incluyendo los mismos deícticos.

En KEN encontramos una serie de combinaciones gramaticales, más bien conservadoras, de los deícticos continuos. En total se hallaron ocho tipos de combinaciones con los deícticos continuos, estas fueron encontradas a principio como en (14) o final de oración como se muestra en (15).

(14) a. ela' tu chicha

$=e \quad-1 \quad-a^{\prime} \quad-$ túul chicha

DEI -EP -ENCL CLAS SUST.chicha

'aquí está una chicha'9 [KEN, 2;04]

b. ela' muñeca, muñeca

e $\quad-1 \quad-a^{\prime} \quad$ muñeca muñeca

DEI -EP -ENCL SUST.muñeca SUST.muñeca

'ahí está muñeca, muñeca' [KEN, 2;04]

(15) a. aa Santa Cos, Santa Cos, beya', beya'

=aa Santa C(l)os, Santa C(1)os, bey -a', bey -a'

$\begin{array}{llllll}\text { INTERJ NP NP } & \text { DEI } & \text {-ENCL DEI } & \text {-ENCL }\end{array}$

'aa Santa Clos, Santa Clos así, asî́ [Ken, 2;04]

b. in pintar te'la'?

in pintar te' $-1 \quad-a^{\prime}$

ERG.1.SG VBO.pintar DEI -EP -ENCL

‘¿pinto aquí?' [KEN, 2;04]

9 Vocabulario utilizado en interacción con la madre, según informe de esta.

Estudios de Lingüística Aplicada, año 35, número 66, diciembre de 2017, pp. 41-75

doi: 10.22201/enallt.01852647p.2017.66.682 
A principio de oración KEN utilizó únicamente las aféresis, como se puede ver en (14a) y (14b) a finales de oración utilizó casi siempre el modal, como en (15a) y en una ocasión también el adverbio locativo te'la', reproducido en (15b).

Lo que resulta muy interesante en la producción de KEN es que no marca la frase nominal. Se registran ya sea la base como se ve en (16) o el enclítico como se ve en (17), ambos enfatizados con negritas. Presentamos los ejemplos registrados para cada caso:

(16) e Banca tu met-ik tene tul u chen beyo'

$\begin{array}{llllll}=\mathrm{e} & \left.\mathrm{B}(\mathrm{l}) \mathrm{anca}(-\mathrm{o})^{\prime}\right) & \mathrm{t}(\text { áan}) \mathrm{u} & \text { meet } & \text {-ik } & \text { teen-e } \\ \text { DET } & \text { NP } & \text { ASP.ERG } & \text { VB.hacer } & \text {-INC } & \text { PRO }\end{array}$

tú(u)l u chéen bey -o'

$\begin{array}{lll}\text { CLAS ERG.3SG ADV } & \text { DEI } \\ \text { 'esa Blanca está haciéndome uno así' } & {[\mathrm{KEN}, 2 ; 04]}\end{array}$

(17) saam i mete de kaa'túul-o’

=sáam mee $\mathrm{i}(\mathrm{n})$ - $\mathrm{t}$-e $\mathrm{e}$ kaa' túul -o’ ADV ERG.1.SG VB.hacer -TRA -SUBJ PREP NUM CLAS -ENCL 'ya hiciste de dos' [KEN, 2;04]

También se registra un caso de ausencia total de deícticos requeridos para la gramaticalidad de la frase en el habla adulta, como se observa en (18).

(18) Blanca Mary ch'ak u bola

=(e) Blanca(-o') Mary(-e') (táan u) ch' a

DEI NP-ENCL NP-TOP ASP ERG.3.SG VB.agarrar -INC POS sUST.bola 'esta Blanca está agarrando la pelota de Mary' [KEN, 2;04]

En el caso de DAN $(2 ; 07,29)$ encontramos el mismo comportamiento que en KEN, no parece recurrente la circunclisis dentro de la frase nominal, sino solamente al principio como en (19) o final de oración como en (20). A continuación presentamos los dos ejemplos encontrados en DAN: 
(19) le ch'u

$=$ le $\quad(x) c^{\prime} u(p a l \quad-o ')$

DEI SUST.muchacha -ENCL

'la muchacha' [DAN, 2;07,29]

(20) na'am ich u ba'axela'

$=(\mathrm{mi})$ na'am ich u ba'axel -a'

NEG PREP POS SUST.juguete -ENCL.PROX

'no hay, está dentro de su juguete' [DAN, 2;07,29]

Nótese en (19) y (20) que el niño produce ambos elementos de la construcción discontinua, pero no como parte de un mismo sistema sino de manera aislada.

Un indicio de uso de la circunclisis lo encontramos en combinación con pronombres personales, como en (21).

(21) je'le teena'

je'l -e teen $-a^{\prime}$

DEI -DEI PRO.1.SG -ENCL

‘aquí está el mío’ [DAN, 2;07,29]

Tanto KEN como DAN participaron en un ejercicio de elicitación de oraciones (Espinosa Ochoa, 2007) para comprobar si habían adquirido la categoría de los determinantes (véase Espinosa Ochoa, 2007) y ambos obtuvieron resultados muy similares. De 12 oraciones repetidas por KEN, incluye las bases solo en cuatro de ellas; DAN repite 7 oraciones y ninguna base. Esto nos refuerza el argumento de que estos niños no han adquirido esta característica gramatical, pero se encuentra en el proceso de incorporación.

\section{3. Última etapa analizada. FEL $(2 ; 10-3 ; 05)$}

Ahora presentamos el análisis realizado para los datos de FEL desde 2;10 hasta 3;05. En el criterio de selección de las emisiones de FEL para este análisis no se tomaron en cuenta los deícticos aislados y se omitieron aquellas combinaciones gramaticales que ya habían sido registradas con anterioridad para los otros tres 
niños. A partir de los 3;01, solo se contemplaron oraciones mayores a MLU 3. ${ }^{10}$ Se mencionan aquí únicamente las construcciones lingüísticas no registradas en los niños más pequeños (ARM, KEN y DAN, véase arriba) consideradas, por tanto, avances en el proceso de incorporación de la circunclisis. ${ }^{11}$ Entre las características encontradas en FEL a los 2;10,07 y ausente en los datos anteriores, documentamos en (22) el primer indicio de la circunclisis en una frase nominal en que aparece el plural.

(22) le telo'ob-a'

le te $-1 \quad-o^{\prime}$ ob $\quad-a$ '

DEI DEI -EP -PL $\quad$-ENCL

'éstos de aquí' [FEL, 2;10,07]

Encontramos también el uso de la discontinuidad con pronombres en el ejemplo (23), como ya se había documentado también con DAN (2;07), e incluso una combinación con sustantivos en el ejemplo (24):

(23) je'leecho'

je' -1 -eech $-\mathrm{o}^{\prime}$

BASE -EP -PRO.ABS.2SG -ENCL

'Ahí estás tú' [FEL, 2;10,07]

(24) ay je' le makinoo'

$\begin{array}{lllll}\text { ay } & \text { je' } & \text { le } & \text { makino } & \text {-o' } \\ \text { INTERJ } & \text { DEI } & \text { DEI } & \text { SUST.máquina } & \text {-ENCL } \\ \text { 'ay, ahí está la máquina' } & \text { [FEL, 2;10,07] }\end{array}$

En esta misma toma de las emisiones de FEL se observa que la ausencia tanto de la base en el ejemplo (25) como del enclítico en el ejemplo (26) sigue patente:

${ }^{10}$ MLU son las siglas de mean length of utterance ('longitud media de emisión').

11 Aunque a los 2;08 FEL no comenzó a hablar de manera espontánea para la recolección de datos de este estudio, a esta edad se le aplicó la prueba de elicitación referida arriba (Espinosa Ochoa, 2007). FEL (2;08) repitió 14 oraciones y solamente 6 bases.

Estudios de Lingüística Aplicada, año 35, número 66, diciembre de 2017, pp. 41-75 doi: 10.22201/enallt.01852647p.2017.66.682 
(25) teen-o' le te'la'

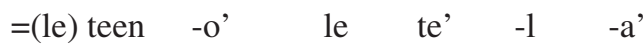

PRO.1.SG -ENCL DEI DEI -EP -ENCL

'Ése soy yo, éste de aquî' [FEL, 2;10,07]

(26) jelu nuuxi ook

jel u nuuxi ook(-o')

DEI ERG ADJ.grande SUST.pie

'Ahí está su pie grande' [FEL, 2;10,07]

A $\operatorname{los} 2 ; 11,13$ las construcciones con circunclisis corresponden a oraciones de una sola frase nominal, como puede verse en los ejemplos reproducidos en (27).

a. je'len tuna'

je' -1 -en tun $-a^{\prime}$

DEI -EP -ABS.1SG ADV -ENCL

'ahí estoy yo entonces' [FEL, 2;11,13]

b. kuxe Tino-e'*12

kux -e Tino -e'

CONJ -DEI NP -TOP

‘y Tino?’ [FEL, 2;11,13]

c. ele muuno'

$=(\mathrm{j}) \mathrm{e}^{\prime} \quad-1 \quad-\mathrm{e} \quad$ muun $\quad-\mathrm{o}^{\prime}$

DEI -EP -DEI ADJ.tierno -ENCL

‘ahí está el (fruto) tierno’ [FEL, 2;11,13]

A partir de los 3;00 ya podemos percibir un uso de la frase nominal como puede observarse en (28).

(28) a. teen in k'áat in xep-e ba'alo'

$\begin{array}{lllllll}=\text { teen(-e') in } & \text { k'áat } & \text { in } & \text { xéep' } & \text { (l)e } & \text { ba'al } & \text {-o' } \\ \text { PRO(-TOP) } & \text { ERG.1.SG } & \text { VB.querer } & \text { ERG.1.SG } & \text { VB.pellizcar DEI } & \text { SUST.cosa } & \text {-ENCL }\end{array}$

${ }^{12}$ Esta combinación de base con el enclítico topicalizador - $e$, , no es gramatical en el habla adulta. 
'Quiero pellizcar esa cosa' [FEL, 3;00]

b. el que tabaja le nuuxi ba'ala' le payaso yaan te'la' $=e l$ que $t(r) a b a j a$ le nuuxi ba'al $-a^{\prime}$ le ART CONJ VB.trabaja-3.SG. DEI ADJ.grande sUST.cosa -ENCL DEI payaso yaan te' 1 a' SUST.payaso EXI.estar DEI -EP -ENCL 'el que trabaja la cosota, el payaso está aquí' [FEL, 3;01,00]

Aunque la última toma de FEL la tenemos a los 3;05, registramos la oración más compleja a los 3;03 en el que se hace patente el dominio de la discontinuidad, como se aprecia en el ejemplo (29).

(29) laaxe [ba] [e biix u-y-oko te'l-a'] k-u-y-oko [te mak-a']

\begin{tabular}{|c|c|c|c|c|c|c|c|c|c|}
\hline laaxe & ba'al & -e & biix & & $-y$ & -ok-o & te' & -1 & -a' \\
\hline VB.mirar & [Sust.cosa & {$[-\mathrm{DEI}$} & ADV & ERG & -EP & -VB.entrar & DEI & $-E P$ & -ENCL] \\
\hline u $\quad-y$ & & & & & & $-a^{\prime}$ & & & \\
\hline $\mathrm{P} \quad-\mathrm{EF}$ & & VB.entra & r [DF & & UST.ta & apa -ENCL] & & & \\
\hline
\end{tabular}

'Míralo cómo entra aquí, entra aquí en la tapa de aquí' [FEL, 3;03]

\subsection{Esquematización del desarrollo de la circunclisis}

En el análisis del uso de ambos clíticos en cada uno de los niños de diferentes edades se observó un desarrollo gradual hacia el uso de la circunclisis. Algunos usos infantiles son claves para comprender las diferentes fases en el desarrollo y nos permiten, por tanto, determinar etapas en la adquisición de la circunclisis. A continuación se presenta un desglosamiento de las etapas en la construcción de la circunclisis desde determinantes de sustantivos aislados hacia su función como marcadores de la frase nominal dentro de la oración:

a) Primera etapa: uso temprano del deíctico utilizado de forma continua, aislada y, al principio, la mayoría de las veces como aféresis, por ejemplo: 
(30) -e'la'

$=$ je'-ela'(?)

je' $-(1) \quad-a^{\prime}$

DEI -EP -ENCL

'Ahí está'

b) Segunda etapa: combinación de las formas deícticas continuas con otros elementos incluyendo otras formas deícticas continuas.

Otros deícticos: la’je’lo'

(31) la'je'lo'

$=$ lela'je'elo'

le $\quad-(1) \quad-a^{\prime} \quad$ je'e $\quad-1 \quad-$ o' $^{\prime}$

DEI $\quad$-EP $\quad$-ENCL DEI $\quad$-EP $\quad$-ENCL

'este, ahí está'

Con pronombres: lo’je'e'éech

(32) lo'je'e'éech

$=$ je'e 1 o' je' e la' téech

DEI EP $\quad$-ENCL $\quad$ DEI $\quad$-EP $\quad$-ENCL $\quad$ PRO.2.SG

'Ahí está este tú'

Con sustantivos: $l a b a^{\prime} a$

(33) laba'a

$=$ je'e $-1 \quad-a^{\prime} \quad$ ba'a

DEI -EP -ENCL SUST.cosa

'ahí está cosa'

c) Tercera etapa: el primer indicio de discontinuidad se utiliza en la combinación de los mismos deícticos, por ejemplo: 
(34) ma' le te-o

$=$ ma' le te - '

NEG DEI DEI -ENCL

'No, ese de allá'

En esta etapa también observamos que los componentes (iniciales y finales) deícticos no son concebidos como parte del mismo sistema en su modalidad discontinua, los niños parecen estar detectando solo el principio o el final de los componentes deícticos.

d) Cuarta etapa: las combinaciones comienzan a ser utilizadas con otras categorías gramaticales.

Con adjetivo: ki e ba'a

(35) ki e ba

= Kúiki'iki le ba'alo'

Kúiki'iki le ba'al -o'

ADJ.sabrosa DEI SUST.cosa -ENCL

"Está sabrosa esa cosa"

e) Quinta etapa: paulatina adición de elementos con uso de los deícticos continuos y uso de la discontinuidad con pronombres personales, por ejemplo:

(36) in pintar te'la'?

in pintar te' -1 -a'?

ERG.1.SG VB.pintar. DEI -EP -ENCL

'pinto aquí'

(37) je'ele teen-a'

je'l -e teen $-a^{\prime}$

DEI -DEI PRO.1.SG -ENCL

'aquí está el mío'

Estudios de Lingüística Aplicada, año 35, número 66, diciembre de 2017, pp. 41-75

doi: 10.22201/enallt.01852647p.2017.66.682 
f) Sexta etapa: marca alternativamente ya sea la base o el enclítico.

Enclítico: saam i mete de kaa'túul-o'

(38) tsaam i mete de kaa'túul-o'

=sáam i(n) mee -t $\quad$-e de kaa' túul -o'

ADV ERG.1.SG VB.hacer -TRA -SUBJ PREP NUM CLAS -ENCL

'ya hiciste de dos'

La base: $\boldsymbol{e}$ Banca tu met-ik tene tul u chen beyo'

(39) e Banca tu met-ik tene tul u chen beyo'

=e $\mathrm{B}(\mathrm{l})$ anca (-o') $\mathrm{t}($ áan) $\mathrm{u}$ meet $-\mathrm{ik}$ teen $-\mathrm{e}$ tú(u)l u chéen bey -o'

DEI NP ASP.ERG VB.hacer -INC PRO CLAS ERG.3SG ADV DEI

'Esa Blanca está haciéndome uno así'

g) Séptima etapa: se incorporan ambos elementos como circunclíticos en oraciones simples, por ejemplo:

(40) Leti'-ob-a' leti' lo

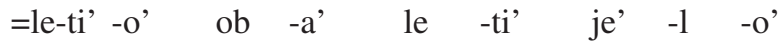

DEI -ENCL - PL - ENCL DEI - ENCL DEI

'El de ellos, es ese'

(41) Ay je le maquino-o'

ay je le maquino -o'

INTERJ DEI DEI SUST.máquina -ENCL

'ay aquí ahí está esa máquina'

h) Octava etapa: se añaden estas estructuras en oraciones más complejas, adquiriéndose así el uso adulto de la circunclisis como marcadora de la frase nominal, por ejemplo: 
(42) el que t(r)abaja le nuuxi baal-a' le payasho yaan te'la'

el que t(r)abaja le nuuxi ba'al -a' le

ART CONJ VB.trabaja-3.sG. DEI ADJ.grande SUST.cosa -ENCL DEI

payaso yaan te' 1 a'

SUST.payaso EXI.estar DEI -EP -ENCL

'el que trabaja la cosota, el payaso está aquí'

\subsection{La influencia del habla adulta}

Tanto las bases como los enclíticos son omitidos en el habla adulta del maya yucateco, esta omisión es común y natural en el habla espontánea. Realizamos un análisis del input para averiguar qué tan frecuentes resultan estas omisiones y de este modo comprobar si tienen algún impacto en la tendencia infantil de atender predominantemente los enclíticos. Utilizamos para este análisis los datos longitudinales de Campamento Hidalgo por ser los más abundantes. Se tomaron en cuenta tanto las emisiones de la mamá (MAM) como las de FLo (7;00), la hermana mayor de FEL y VAL, ya que dentro de la familia tiene una función particular como cuidadora. En ausencia de un adulto, FLO es la que toma decisiones y da órdenes a los niños, su rol dentro de la familia, e incluso de manera cultural, está muy bien establecido. Por esta razón, aunque flo es aún una niña, consideramos que su habla pudiera tener una influencia importante en el habla de sus hermanos. Se contaron todas las emisiones en las que MAM y FLO emitieron una base o un enclítico dentro de una frase nominal, es decir, cada vez que los deícticos locativos fueron utilizados en su característica de discontinuos. Las oraciones se clasificaron de la siguiente manera:

i) Uso discontinuo completo: cuando aparece tanto la base con la lateral ' $l$ ' como el enclítico en su modalidad estandarizada.

ii) Uso discontinuo incompleto: aunque aparece tanto la base como el enclítico, el primer fonema de la base está elidido. Esta base es casi siempre el demostrativo sin la lateral, como en el ejemplo (43). 
(43) Ba'axe beyo'?

Baax -e bey -o'

PRO.INT.que -DEI DEI -ENCL

¿Qué es eso?'

en que aparece como clítico de la palabra anterior, aunque modifique a la posterior. En vez de aparecer como en (44).

(44) Ba'ax le beyo'?

Baax $\quad-1 \quad-e$ bey -0 '

PRO.INT.que -EP -DEI DEI -ENCL

¿Qué es eso?'

El único otro caso en que el primer fonema de la base está elidido es el del ostensivo evidencial, como se muestra en (45).

(45) ele pek'o'

$=(j)$ 'le pek'o'

je' $-1 \quad$-e $\quad$ pek'

DEI -EP -DEI SUST.perro -ENCL

'Allá está ese perro'

iii) Omite la base o la asimila con el imperativo, como en (46).

(46) taase baalo'

taas -e(j) (l)e ba'al -o'

VB.traer -IMP SUST.cosa -ENCL

'trae la cosa (tráelo)'

iv) El uso de la discontinuidad completa. En este rubro se tomaron en cuenta emisiones gramaticales de combinación discontinua entre deícticos, en que aparece una base suelta que se completa con el enclítico de la base siguiente, como en (47). 
(47) je le ba'alo'

je le ba'al -o'

DEI le SUST.cosa -ENCL

'Ahí está esa cosa'

Los resultados obtenidos se resumen en el Cuadro 3.

CuAdRo 3. Porcentaje de emisiones para cada tipo de uso de enclíticos en el habla adulta

\begin{tabular}{|c|c|c|c|c|}
\hline \multirow{2}{*}{ Usos } & \multicolumn{2}{|c|}{ MAM } & \multicolumn{2}{|c|}{ FLO } \\
\hline & Ocurrencias & $\%$ & Ocurrencias & $\%$ \\
\hline Uso discontinuo completo & 12 & 24.4 & 35 & 28 \\
\hline Uso discontinuo incompleto & 10 & 20.4 & 27 & 21 \\
\hline Asimilación/Omisión de la base & 24 & 49 & 46 & 36 \\
\hline Uso discontinuo completo & 3 & 6.2 & 19 & 15 \\
\hline Totales & 49 & 100 & 127 & 100 \\
\hline
\end{tabular}

Vemos que tanto en el habla de MAM como en el de FLO, lo más común es encontrar la base asimilada con la terminación - ej del imperativo o bien, omitida. La presencia de las bases en el input no presenta un patrón estable, ya que se asimilan con el imperativo. Esto también puede estar influyendo para que la tendencia más fuerte de los niños al incorporar la circunclisis, sea principalmente mediante el uso de los enclíticos.

\section{Discusión}

De acuerdo con nuestros resultados podemos inferir que ambas posiciones de los clíticos son relevantes para la circunclisis: principio y final de palabra, frase y oración de manera independiente. La función sintáctica de ambos elementos no es tan relevante como la forma, para que los niños adquieran esta categoría como un conjunto íntegro que exprese determinación de la frase nominal se requieren dos años de uso continuo. ¿A qué se puede deber este fenómeno? Es posible atribuir este fenómeno a la lengua que los niños escuchan; en el habla espontánea se utilizan algunas veces solo las bases y la mayoría de las veces 
solo los enclíticos (o la base aparece asimilada con la desinencia del imperativo). Es posible entonces que la falta de homogeneidad en el input esté llevando a los niños a generar diferentes hipótesis frente a este fenómeno y les impida concebirlo como una totalidad desde un principio, con una tendencia mayor hacia el lado derecho de la oración. Nuestros resultados apoyan la tendencia más fuerte en la adquisición infantil del maya hacia la adquisición morfológica a la derecha de palabra u oración (Pye et al., 2007). Recordemos además que el maya yucateco es una lengua en la que sus constituyentes en general se ramifican o extienden hacia la derecha: "the subject follows the predicate, the nominal and adverbial dependants follow the verb, attributes follow their head noun [...] adpositions are prepositions, dependant clauses follow their matrix clause" (Lehmann, 1998: 22). ${ }^{13}$ Mateo (2015) documenta que en la adquisición del maya q'anjob'al los niños muestran dominio temprano de los morfemas flexivos que se combinan con verbos cuya inicial es una vocal y se omiten con más frecuencia si se combinan con verbos cuya inicial es una consonante. Mateo realiza un estudio en el que prueba que este fenómeno se debe a que los niños son sensibles al patrón fonológico cvc de esta lengua maya. Sabemos que los niños mayas yucatecos omiten frecuentemente la base en posición final de oración (Espinosa Ochoa, 2007). Es posible entonces que la omisión, ligada con la adquisición tardía de la base en esta posición, se deba también al patrón consonántico del yucateco. Es interesante notar que al revisar los resultados de la prueba de elicitación, la gran mayoría de las omisiones se deben a que los niños tienen una tendencia a seguir el patrón CVC, aunque sería necesario un estudio más riguroso para una afirmación más contundente a este respecto.

Podemos concluir entonces que los deícticos continuos y discontinuos no están relacionados en la adquisición, pues en la discontinuidad los niños no usan ambos elementos sino hasta los 3;00. En una primera etapa $(1 ; 03-1 ; 07)$, muy incipiente, ARM emite ambos elementos de la circunclisis independientemente acompañados de una palabra, las razones por las que esto sucede requieren todavía investigación y reflexión. En una segunda etapa (2;03-2;07), DAN y KEN

13 “[E]l sujeto va detrás del predicado, los dependientes nominales y adverbiales van detrás del verbo, los atributos van detrás de su sustantivo principal [...] las adposiciones son preposiciones, las cláusulas dependientes van detrás de su matriz." 
emiten ambos elementos por separado en frases y palabras, pero con mayor tendencia a eliminar la base (de lado izquierdo) y eliminando siempre la base en posición media cuando se trata de oraciones más largas. Esto puede explicarse nuevamente por la tendencia infantil de las lenguas mayas a atender el lado derecho de palabra y oración (Pye et al., 2007). Solamente FEL (2;10-3;08) utilizó ambos elementos de la circunclisis por encontrarse en una etapa más avanzada en el proceso de adquisición.

Desde un ángulo constructivista (Lieven et al., 2003) podemos decir que la adquisición de la circunclisis consiste en una operación sumatoria de elementos. Encontramos que requiere de cuatro etapas importantes, pues no se reconoce en un principio el sistema discontinuo como una unidad, sino como elementos independientes. Las construcciones nominales toman forma independientemente a principio y final de oración, para situarse finalmente como una unidad cuando los elementos anteriores ya están consolidados. Cada etapa se diferenció de la otra porque se encontraron características diferentes de uso de los morfemas en el habla infantil, mostrando un desarrollo paulatino. En la primera etapa las palabras y/o frases nominales simples son rodeadas ya sea por la base o por el enclítico, con mayor tendencia al uso del último. En la segunda etapa se forman oraciones más complejas rodeadas ya sea por la base o por el enclítico. La frase nominal a inicio de oración carece del enclítico y al final de oración carece de la base. En la tercera etapa el niño comienza a utilizar ambos elementos en palabras y frases nominales. Y, finalmente, en la cuarta etapa es capaz de utilizar los clíticos a la manera adulta, flanqueando la frase nominal. Esto sugiere que la adquisición de elementos sintácticos requiere una construcción paulatina de los elementos que la conforman y que la función sintáctica como determinantes no genera la gramática adulta desde un principio, es decir, la categoría de determinante no está predeterminada en la mente del niño, tampoco el fenómeno de la circunclisis: se necesita una evolución gradual en el proceso de la adquisición de la lengua materna que equivale a una suma de elementos.

En el maya yucateco se requiere de un proceso de construcción primaria de palabras complejas hacia oraciones complejas (Aksu Koç \& Slobin, 1985). Encontramos también que tanto en verbos (Pye et al., 2007) como en sustantivos existe la tendencia a anexar los morfemas hacia el lado derecho de palabra y oración. Sugerimos que esto puede deberse a las características tanto de la 
lengua como del habla, ya que no presentan un patrón consistente. En el habla espontánea se puede llegar a omitir la base o el enclítico, con mayor frecuencia del primero que el segundo. La otra posible causa recae en la característica prosódica de la lengua, acentuada en el topicalizador, pero esto es un tema pendiente para futuras investigaciones.

\section{REFERENCIAS}

Aksu Koç, Ayhan, \& Slobin, Dan (1985). The acquisition of Turkish. En Dan Isaac Slobin (Ed.), The crosslinguistic study of language acquisition (pp. 839-880). Hillsdale: Lawrence Erlbaum.

Bohnemeyer, Jürgen, \& Stolz, Christel (2006). Spatial reference in Yukatek Maya: A survey. En Stephen C. Levinson \& David P. Wilkins (Eds.), Grammars of space: Explorations in cognitive diversity (pp. 273-310). Cambridge: Cambridge University Press.

Brown, Penelope (1997). Isolating the cvc root in Tzeltal Mayan: A study of children's first verbs. En Eve V. Clark (Ed.), The proceedings of the Twenty-Eighth Annual Child Language Research Forum (pp. 41-52). Stanford: Center for the Study of Language and Information Publications.

Brown, Penelope (1998). Children's first verbs in Tzeltal: Evidence for an early verb category. Linguistics, 36(4), 713-753.

Bybee, JoAn (1995). Regular morphology and the lexicon. Language and Cognitive Processes, $5(10), 425-455$.

Carrillo Carreón, Carlos Alfonso (2005). La adquisición de los pronombres de referencia cruzada en el maya yucateco. Estudio de caso (Tesis de maestría inédita). Universidad Autónoma de Yucatán, Mérida.

De León, Lourdes (1999). Verb roots and caregiver speech in early Tzotzil (Mayan) acquisition.

En Barbara A. Fox, Dan Jurafsky \& Laura A. Michaelis (Eds.), Cognition and function in language (pp. 99-119). Stanford: Center for the Study of Language and Information Publications.

De León, Lourdes (2012). Language socialization and multiparty participation frameworks. En Alessandro Duranti, Elinor Ochs \& Bambi B. Schieffelin (Eds.), The handbook of language socialization (pp. 81-112). Malden: Wiley-Blackwell.

Estudios de Lingüística Aplicada, año 35, número 66, diciembre de 2017, pp. 41-75

doi: 10.22201/enallt.01852647p.2017.66.682 
EsPINOSA OCHOA, MARY Rosa (2002). Los pronombres demostrativos en la adquisición temprana del español como lengua materna (Tesis de licenciatura). Universidad Nacional Autónoma de México, México.

Espinosa OchOA, Mary Rosa (2007). The early acquisition of determiners in Yucatec Maya and Spanish. En Naomi Hilton, Rachel Arscott, Katherine Barden, Arti Krishna, Sheena Shah \& Meg Zellers (eds.), CamLing 2007. Proceedings of the Fifth University of Cambridge Postgraduate Conference in Language Research (pp. 56-63). Cambridge: Universidad de Cambridge.

Espinosa Ochoa, Mary Rosa (2011). Dame las piezas y lo armo: las primeras preguntas y la influencia del habla dirigida al niño. En Cecilia Rojas Nieto \& Donna Jackson-Maldonado (eds.), Interacción y uso lingüístico en el desarrollo de la lengua materna (pp. 92-115). México: Instituto de Investigaciones Filológicas, Universidad Nacional Autónoma de México/Universidad de Querétaro..

Flores Vera, Miguel Ángel (1998). La adquisición temprana del maya yucateco (Tesis de licenciatura inédita). Universidad Autónoma de Yucatán, Mérida.

Gutiérrez Bravo, Rodrigo (2015). Las cláusulas relativas en maya yucateco. México: El Colegio de México.

Hanks, William (1990). Referential practice: Language and lived space among the Mayas. Chicago: Chicago University Press.

Johnson, CAROLYn (1983). The development of children's interrogatives: From formulas to rules. Papers \& Reports on Child Language Development, 22, 108-115.

Karmiloff Smith, Annette (1979). A functional approach to child language: A study of determiners and reference. Cambridge: Cambridge University Press.

Lehmann, Christian (1998). Possession in Yucatec Maya. Unterschleissheim: Lincom Europa.

Lieven, Elena (2008). Learning the English auxiliary: A usage-based approach. En Heike Behrens (Ed.), Corpora in language acquisition research: History, methods, perspectives (pp. 61-98). Ámsterdam: John Benjamins.

Lieven, Elena; Behrens, Heike; Speares, Jennifer, \& Tomasello, Michael (2003). Early syntactic creativity: A usage-based approach. Journal of Child Language, 30(2), 333-370.

Mateo, Pedro (2015). The acquisition of inflection in Q'anjob'al Maya. Ámsterdam: John Benjamins. doi: 10.1075/tilar.14

Monforte y MAdera, Jorge (1999). Estructura y función del determinante le...á, le...ó, le...é en el maya yucateco: un estudio empírico descriptivo (Tesis de maestría inédita). Centro de Investigaciones y Estudios Superiores en Antropología Social, México.

Estudios de Lingüística Aplicada, año 35, número 66, diciembre de 2017, pp. 41-75 doi: 10.22201/enallt.01852647p.2017.66.682 
Pfeiler, Barbara (2002). Noun and verb acquisition in Yucatec Maya. En Maria D. Voeikova \& Wolfgang U. Dressler (Eds.), Pre- and protomorphology: Early phases of morphological development in nouns and verbs (pp. 75-82). Múnich: LINCOM Europa.

Pfeiler, BARbara (2007). Lo oye, lo repite y lo piensa. La contribución de instrucciones explícitas a la adquisición del maya yucateco. En Barbara Pfeiler (Ed.), Learning indigenous languages: Child language acquisition in Mesoamerica (pp. 183-202). Berlín: De Gruyter Mouton.

Pfeiler, Barbara, \& Briceño, Martín (1997). Early verb inflection in Yucatec Maya. Papers and Studies in Contrastive Linguistics, 33, 117-125.

Pine, Julian, \& Lieven, Elena (1997). Slot and frame patterns in the development of the determiner category. Applied Psycholinguistics, 18(2), 123-138.

Pine, Julian, \& Martindale, Helen (1996). Syntactic categories in the speech of young children: The case of the determiner. Journal of Child Language, 23(2), 369-395.

Pye, Clifton (1983). Mayan telegraphese: Intonational determinants of inflectional development in Quiche' Mayan. Language, 59(3), 583-604.

Pye, Clifton (1992). The acquisition of K'iche' Maya. En Dan Isaac Slobin (Ed.), The crosslinguistic study of language acquisition (pp. 221-308). Hillsdale: Lawrence Erlbaum.

Pye, Clifton; Pfeiler, Barbara; De león, lourdes; Brown, Penelope, \& Mateo, Pedro (2007). Roots or edges? Explaining variation in children's early verb forms across five Mayan languages. En Barbara Pfeiler (Ed.), Learning indigenous languages: Child language acquisition in Mesoamerica (pp. 15-46). Berlín: De Gruyter Mouton.

Theakston, Anna; Lieven, Elena; Pine, Julian, \& Rowland, Caroline (2002). Going, going, gone: The acquisition of the verb 'go'. Journal of Child Language, 29(4), 783-811. doi:10.1017/S030500090200538X

Tomasello, Michael (2003). Constructing a language: A usage-based theory of language acquisition. Cambridge: Harvard University Press.

Estudios de Lingüística Aplicada, año 35, número 66, diciembre de 2017, pp. 41-75

doi: 10.22201/enallt.01852647p.2017.66.682 


\section{ANEXo}

Abreviatura de las glosas

$\begin{array}{llll}\text { ART } & \text { Artículo } & \text { INTERJ } & \text { Interjección } \\ \text { ABS } & \text { Absolutivo } & \text { NEG } & \text { Negación } \\ \text { ADV } & \text { Adverbio } & \text { NP } & \text { Nombre propio } \\ \text { ADJ } & \text { Adjetivo } & \text { NUM } & \text { Numeral } \\ \text { ASP } & \text { Aspecto } & \text { POS } & \text { Posesivo } \\ \text { CLAS } & \text { Clasificador } & \text { PL } & \text { Plural } \\ \text { CONJ } & \text { Conjunción } & \text { PREP } & \text { Preposición } \\ \text { DEI } & \text { Deíctico } & \text { PRO } & \text { Pronombre personal } \\ \text { DET } & \text { Determinante } & \text { PROX } & \text { Próximo } \\ \text { DIST } & \text { Distal } & \text { SG } & \text { Singular } \\ \text { ENCL } & \text { Enclítico } & \text { SUBJ } & \text { Subjuntivo } \\ \text { EP } & \text { Epéntesis } & \text { SUST } & \text { Sustantivo } \\ \text { ERG } & \text { Ergativo } & \text { TRA } & \text { Transitivizador } \\ \text { EXI } & \text { Existencial } & \text { TOP } & \text { Topicalizador } \\ \text { FEM } & \text { Femenino } & \text { VB } & \text { Verbo } \\ \text { IMP } & \text { Imperativo } & & 1 \text { Primera persona gramatical } \\ \text { INC } & \text { Incompletivo } & & 2 \text { Segunda persona gramatical } \\ \text { INT } & \text { Interrogativo } & & 3 \text { Tercera persona gramatical }\end{array}$


